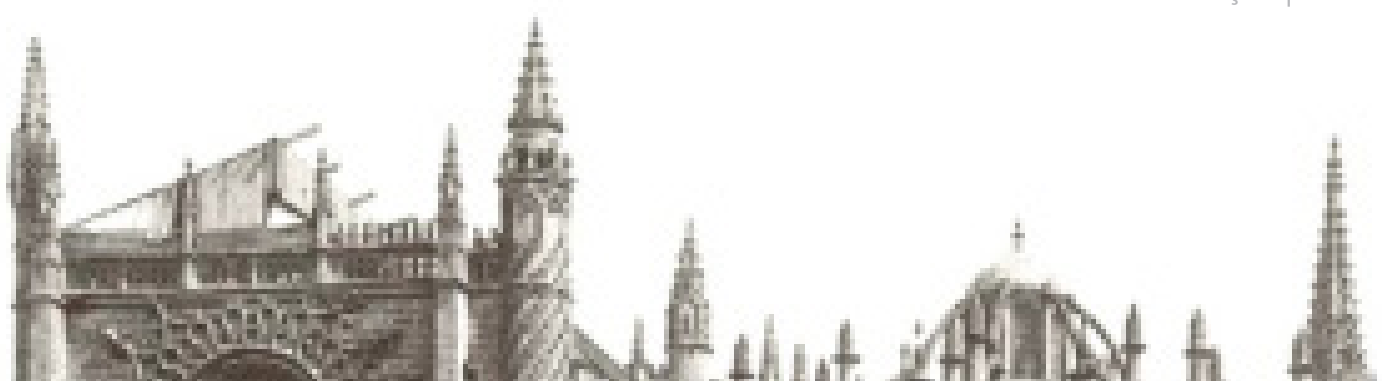

\title{
Coberturas sostenibles en excavaciones arqueológicas. Metodología de aplicación al caso de mosaicos en el Conjunto Arqueológico de Itálica (Santiponce, Sevilla)
}

\author{
M. Ordóñez-Martín, J.C. Gómez de Cózar
}

\begin{abstract}
Resumen: Es conocido que uno de los retos del trabajo arqueológico, es el de evitar o reducir los efectos del llamado"trauma de excavación", donde la modificación acelerada de condiciones higrotérmicas, junto al efecto de la radiación solar, altera las condiciones de equilibrio del objeto soterrado. Esto lleva a la necesidad de cobertura de yacimientos arqueológicos durante la excavación, realizada frecuentemente mediante sistemas fijos o desmontables más propios del mundo productivo o industrial, de alta huella ecológica, y con efecto limitado, garantizando en su mayoría únicamente situaciones de sombra. No obstante, entendemos que es posible dar una solución global desde la arquitectura a los numerosos requerimientos del objeto excavado, tales como: necesidad de coberturas con mínimos apoyos, coberturas ligeras de gran adaptabilidad a la evolución de la excavación, limitación de costes, o necesidad de control de las condiciones higrotérmicas del espacio cubierto mediante procedimientos limpios que minimicen el impacto medioambiental.
\end{abstract}

Palabras clave: Sostenibilidad, conservación preventiva, coberturas activas, excavación arqueológica, diseño paramétrico, control higrotérmico

\section{Sustainable coverage in archaeological excavations. Methodology of application to the case of mosaics in the Archaeological Ensemble of Italica (Santiponce, Sevilla)}

Abstract: One of the challenges of the archaeological work is to prevent or reduce the effects of the "excavation trauma", where the accelerated changes of hygrothermal conditions together with the effect of solar radiation, alter the equilibrium conditions of the buried object. This leads to the need for a coverage during excavation of archaeological sites which is most often performed by fixed or removable systems that come from the productive or industrial world. They have high ecological footprint and limited impact, and ensure, mostly, only shady situations. But we understand that it is possible to provide a global solution from architecture to the numerous requirements of the excavated object, such as: covering systems with minimal supports, light covers highly adaptable to excavation changes, limited budget, or the control of hygrothermal conditions of the covered space through clean procedures that minimize environmental impact.

Keywords: Sustainability, preventive conservation, active coverages, archaeological excavation, parametric design, hygrothermal control

\section{Introducción}

Es habitual entender la arquitectura de rápido montaje, como elemento accesorio en las intervenciones patrimoniales, en situaciones transitorias, como "medio auxiliar", por su cualidad de ligereza o poca materialidad. Esta visión, hace que la convivencia de esta arquitectura con el bien patrimonial sea a través de una relación de superposición. Nosotros planteamos, sin embargo, que dichas propiedades de: ligereza, transformabilidad y rápida respuesta, permiten superar el concepto de elemento accesorio, y convertirse en elementos complementarios al objeto a proteger, especialmente en situaciones transitorias, frecuentes en la actividad arqueológica. Esto puede lograrse gracias a sus cualidades de fácil desmontabilidad, reciclabilidad y escasa huella (Gómez de Cózar et al. 2019).

Este trabajo, pretende establecer una metodología de intervención asociada a la cobertura de yacimientos arqueológicos, dentro de la conservación preventiva de los mismos, entendiendo esta como la acción de "garantizar la conservación y el mantenimiento de los bienes culturales, aplicando todos los medios posibles externos a los mismos" (Frazzi 2002; 95-111). 
En el caso del objeto excavado, la conservación preventiva supondrá las acciones necesarias para atenuar el "trauma de excavación" (Díaz Martínez 2005; 110-130). Los objetos enterrados, permanecen en un ambiente diferente para el que fueron creados, llegando con el paso del tiempo a una situación de equilibrio con las nuevas condiciones mecánicas, físicas, de exposición a luz, temperatura, humedad, microorganismos, presencia de oxígeno, etc. Al excavarse, quedan expuestos a nuevas condiciones de abundancia de oxígeno, $\mathrm{CO}_{2}$, contaminantes del aire, acidez de lluvias, luz, oscilaciones de temperatura y humedad, etc. Estas nuevas condiciones, suponen un shock para el objeto excavado. Será pues objetivo de la intervención de conservación, evitar al máximo estos procesos de degradación, con medidas que disminuyan los desequilibrios entre el objeto y el nuevo ambiente. (Lacayo 2001;453-457).

La acción de conservación requiere conocer los factores de alteración del objeto excavado, que pueden ser de origen antrópico o ambientales (físicas, químicas o biológicas) (Giles; Bouzas; Pinto 2003).

Desde la arqueología, diversos autores coinciden en que la cobertura es la única actuación que permite la pervivencia de las estructuras y elementos que forman los yacimientos, (Díaz Martínez 2005; 1 19), planteándose los requerimientos de sus coberturas, destacándose los siguientes:

- Intervenciones con mínimos apoyos y grandes luces. - Solución compatible con la evolución de la excavación, realizadas por fases, con sistemas de tipo modular.

- Cubiertas parciales reversibles que supongan soluciones neutras de fácil desmontaje.

- Coberturas que no compitan con los restos a proteger, diferenciándose claramente los añadidos.

- Coberturas que garanticen una protección solar efectiva.

\section{Objetivos de la investigación.}

Teniendo en cuenta que el objetivo primordial de la intervención patrimonial, ha sido la recuperación de la materialidad y los valores del objeto a proteger, a partir de un estudio basado en la metodología pluridisciplinar (Price 1984; Carrera 2018), esta investigación, plantea los siguientes objetivos para establecer una metodología de intervención que permita dar respuesta a los requerimientos de protección de los objetos arqueológicos en proceso de excavación, garantizando unas condiciones de conservación efectivas, evitando los fenómenos de "trauma de excavación" por procesos de: heladicidad, cambios bruscos de temperatura o humedad e incidencia de radiación solar directa, y que permitan abrir dicha intervención, a acciones de interpretación.

Desde el punto de vista especifico, se plantean los siguientes objetivos:
- Uso de la arquitectura ligera y de rápido montaje como elemento no sólo de protección sino de puesta en valor del elemento patrimonial. Tal como se ha indicado anteriormente, los parámetros habituales que definen a este tipo de sistemas (Gómez de Cózar et al. 2017) son: capacidad de adaptación a condiciones de contorno variables, reversibilidad y mínimo impacto medioambiental, idóneos para cubrir yacimientos.

- Control ambiental del espacio cubierto durante su intervención, mediante procedimientos pasivos que no requieran energía o que ésta sea mínima y pueda obtenerse mediante fuentes renovables (Hoyano; Jiang 2009;1119-1127).

- Comprobación de la minimización del impacto medioambiental de la solución propuesta mediante herramientas de Análisis del Ciclo de Vida.

\section{Metodología propuesta.}

Para alcanzar los objetivos que definen esta aportación, se ha diseñado la siguiente metodología original que, correctamente aplicada, permitirá la intervención en cualquier yacimiento arqueológico que necesite salvaguardar una superficie inferior en condiciones higrotérmicas adecuadas:

- Análisis del yacimiento a cubrir atendiendo a la singularidad de sus restos, naturaleza, posición, geometría, planificación de excavación, potencial de lugares del suelo que pueden ser utilizados como apoyos, condiciones de temperatura y humedad anuales y estudio visual de contorno que permita plantear la posibilidad de que la cobertura permanezca una vez terminadas las tareas de excavación y/o puesta en valor.

- Desarrollo de una cubierta ligera y de rápido montaje basada en un sistema espacial de dos capas sobre mínimos apoyos. A partir de las condiciones de contorno establecidas en el punto anterior, mediante software paramétrico, determinando la geometría específica de la cubierta. Las dos capas permiten generar una cámara de aire (controlada) que posibilitará el control higrotérmico del espacio protegido. Comprobación como estructura de la cubierta para optimizar su materialidad.

- Optimización de la cobertura a partir de software de simulación numérico original, que permite simular condiciones ambientales tanto del interior del recinto como de su suelo a partir del control de la ventilación de la cámara de la envolvente.

- Análisis del Ciclo de Vida de la solución planteada, conforme a las normas ISO 14040 (UNE-EN ISO 14040 2006), ISO 14044 (UNE-EN ISO 14044 2006) y EN 15978 (UNE-EN 15978 2012), atendiendo tanto a energía incorporada como a la operacional en categoría de GWP (calentamiento global) con objeto de validar la solución de diseño. En el caso de que el impacto sea excesivo, la solución deberá reconfigurarse (geometría, 


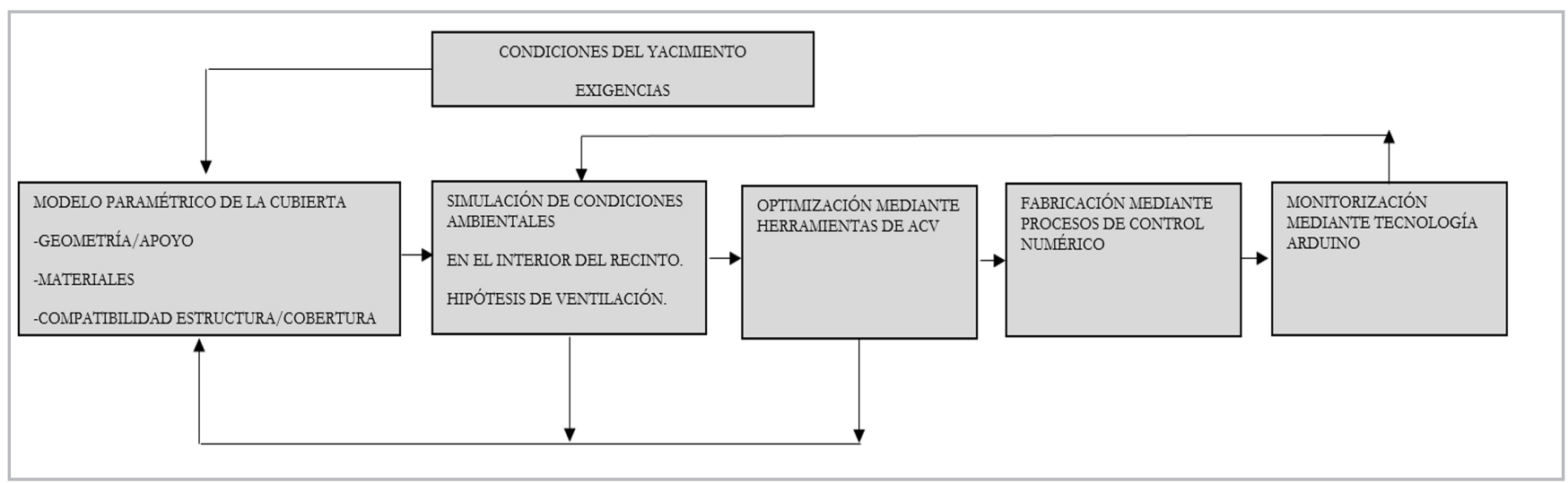

Gráfico 1.- Esquema de la metodología propuesta.

materiales y ciclos de ventilación) para minimizar su impacto.

- Fabricación de la cubierta mediante procedimientos de control numérico, permitiendo un mínimo tiempo de fabricación.

- Control in situ, mediante monitorización basada en tecnología Arduino. De este modo, el modelo numérico de simulación de las condiciones ambientales del recinto podrá calibrarse en función de las condiciones reales del recinto.

Este artículo, desarrolla la metodología descrita atendiendo a los apartados que tienen que ver con la creación paramétrica del modelo, a su simulación numérica y a su comportamiento medioambiental mediante herramientas de ACV. [gráfico 1].

\section{Emplazamiento y estudio de condiciones de contorno}

La aplicación de la metodología de estudio se aplicará como primer modelo, en un yacimiento arqueológico del sur de España, concretamente el Conjunto Arqueológico de Itálica, en la localidad de Santiponce, (Sevilla). Dicho estudio surge de las necesidades de protección de mosaicos en procesos de restauración acometidos por el
Conjunto. El Plan Director del yacimiento (año 2001) prevé en el apartado de mosaicos, ante la escasez de recursos, su cobertura mediante geotextiles y arena, entendiendo que dos de los agentes generadores de mayor deterioro son los cambios de humedad y temperatura. No obstante, aboga por la implantación de cubiertas físicas en altura previo estudio paisajístico.

Por otra parte, se plantea desde el Conjunto, la idoneidad de implantación de un sistema de cobertura experimental temporal, con el fin de contrastar resultados de monitorización con actuales coberturas efímeras instaladas, cubiertas convencionales a base de estructura metálica y materiales plásticos de sombra.

El emplazamiento elegido responde, además, al estudio de las condiciones de contorno (accesibilidad, estado de conservación, perfil climático, etc.), optándose por mosaico perteneciente a la conocida como "Casa de Neptuno".

Se procederá al levantamiento planimétrico del objeto arqueológico y de su entorno, con el fin de diseñar un prototipo que responda a las condiciones de mínima huella en su instalación, así como de absoluta reversibilidad de la intervención [figura 1].

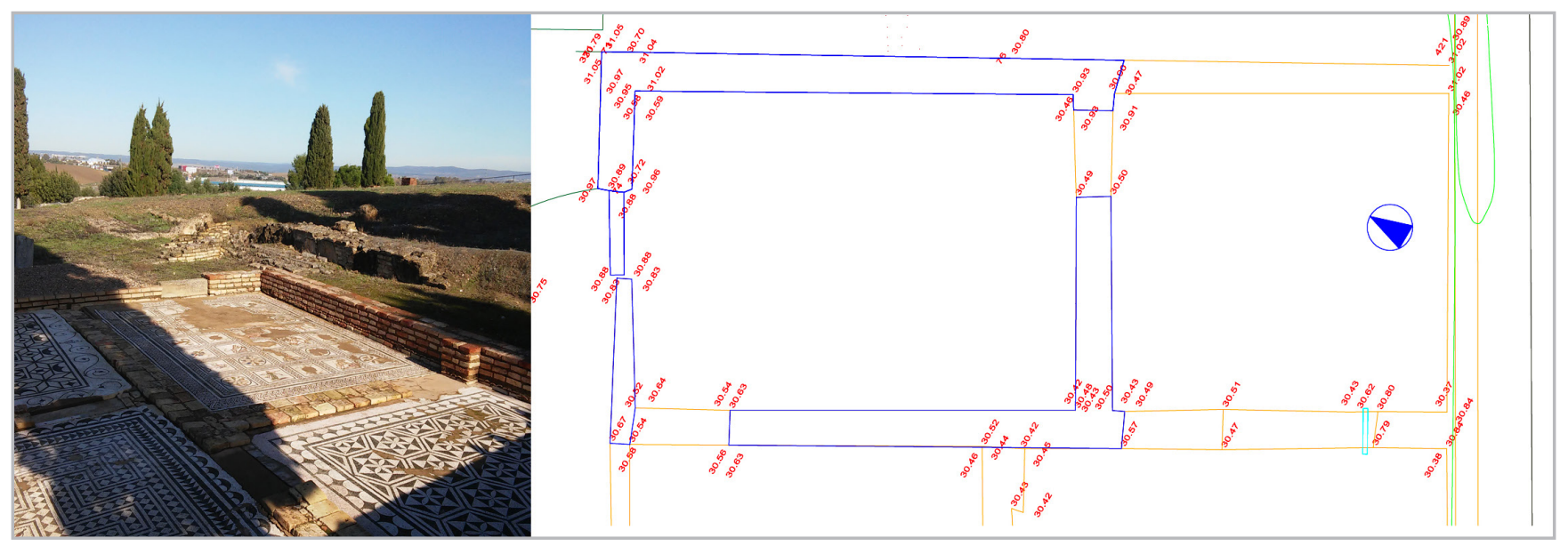

Figura 1.- Fotografía de emplazamiento y levantamiento planimétrico. 
El mosaico elegidoes adyacente a otros tres pertenecientes al mismo conjunto doméstico, y presenta una delimitación irregular en cuanto a la altura de arranques de muros que lo delimitan, fruto de intervenciones de consolidación realizadas. Esta situación, ligada a la topografía irregular de su entorno inmediato, condicionará la generación del modelo de cobertura.

\section{Generación paramétrica de modelo de cubierta, comprobación estructural y fabricación mediante tecnología CNC.}

Una vez definidas las condiciones de contorno, se procede a la generación del modelo geométrico de la cobertura. Para dicha generación, se emplea software original de diseño paramétrico, que permite generar geometrías ilimitadas pudiendo variar en tiempo real los diversos parámetros que las condicionan.

La herramienta de diseño permite actuar sobre los parámetros definitorios del sistema, basado en la "Patente Florín" (Gómez de Cózar; García Diéguez 2001). [figura 2].

Estos son:

- Variación del número de módulos de la malla tridimensional, generando modelos de mayor o menor extensión, adaptándose a la geometría y tamaño del área a proteger.

- Modificación del canto de la malla, lo cual permitirá adecuarse a las necesidades del yacimiento en cuanto a luz máxima entre apoyos, así como a las acciones previstas (peso propio del textil de cobertura, cargas de nieve, etc.).

- Variaciones sobre la curvatura de la malla estructural esférica, permitiendo actuar sobre la estabilidad, limitando las deformaciones, especialmente ante acciones eólicas.

Posteriormente a la generación, será necesaria la comprobación estructural de la malla y el dimensionado de las distintas barras que la componen (rombos, aspas y diagonales).

Igualmente, se deberán dimensionar los apoyos de dicha cubierta, condicionados a la geometría del terreno existente, con la premisa de ejecutar un número mínimo de apoyos, y siempre externos al objeto excavado.

Para dicho dimensionado, se emplea software de cálculo matricial espacial de mallas de barras, desarrollado por Cype Ingenieros S.A. Dicha herramienta, permite el dimensionado de las barras de la malla estructural en limitando los estados límites últimos y de servicio para materiales como acero, aluminio o madera. En el caso de estudio, se fijarán los parámetros de resistencia mecánica relativos a panel de fibras de densidad media (MDF), previendo la ejecución de barras y nudos mediante la combinación de piezas de dicho material, hasta conseguir la sección resistente necesaria. Se chequearán igualmente las deformaciones debidas especialmente a

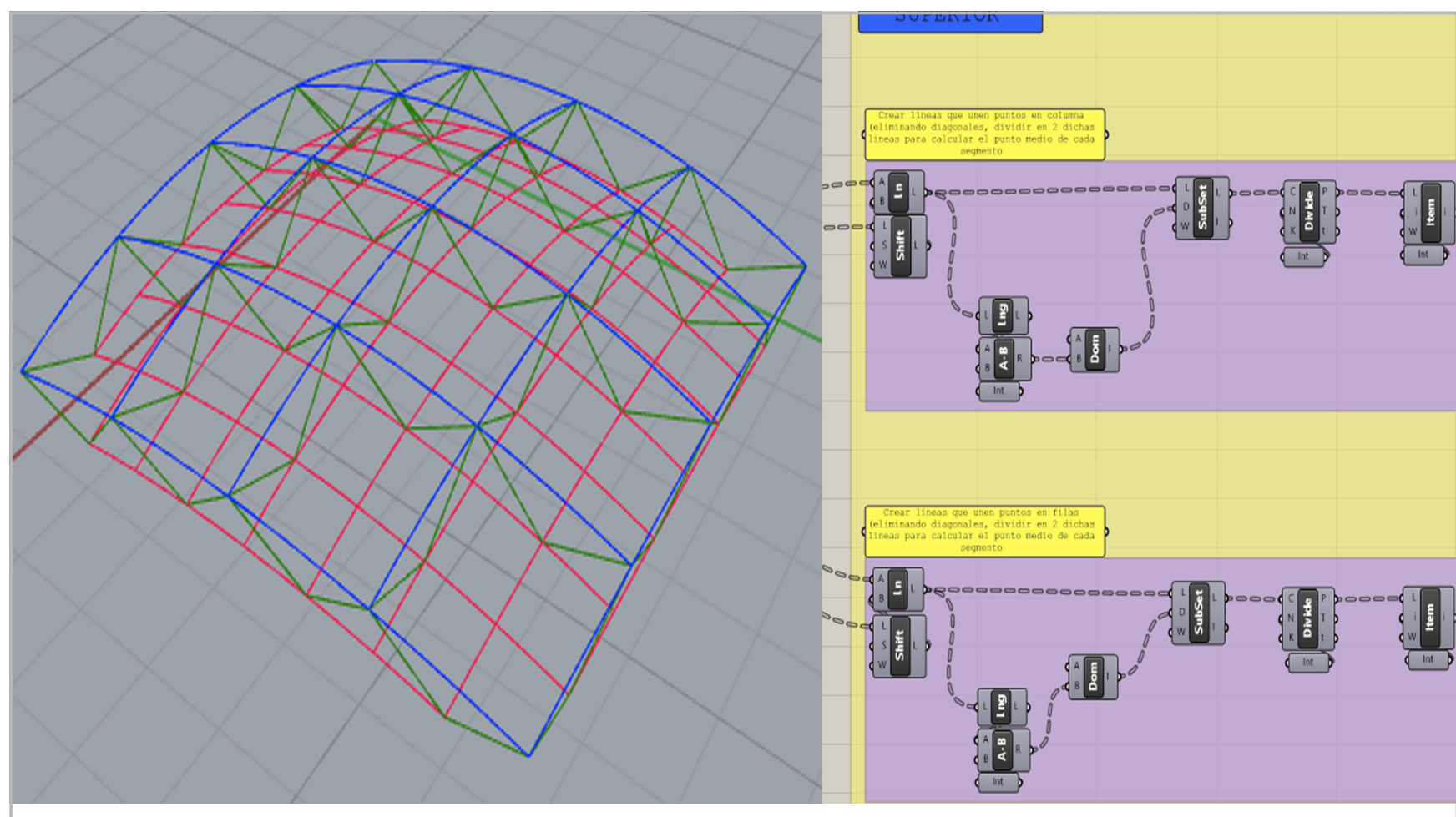

Figura 2.- Pantalla de control de parámetros de generación de la malla estructural, Sistema Florín, de la cubierta mediante herramienta Grasshopper. 


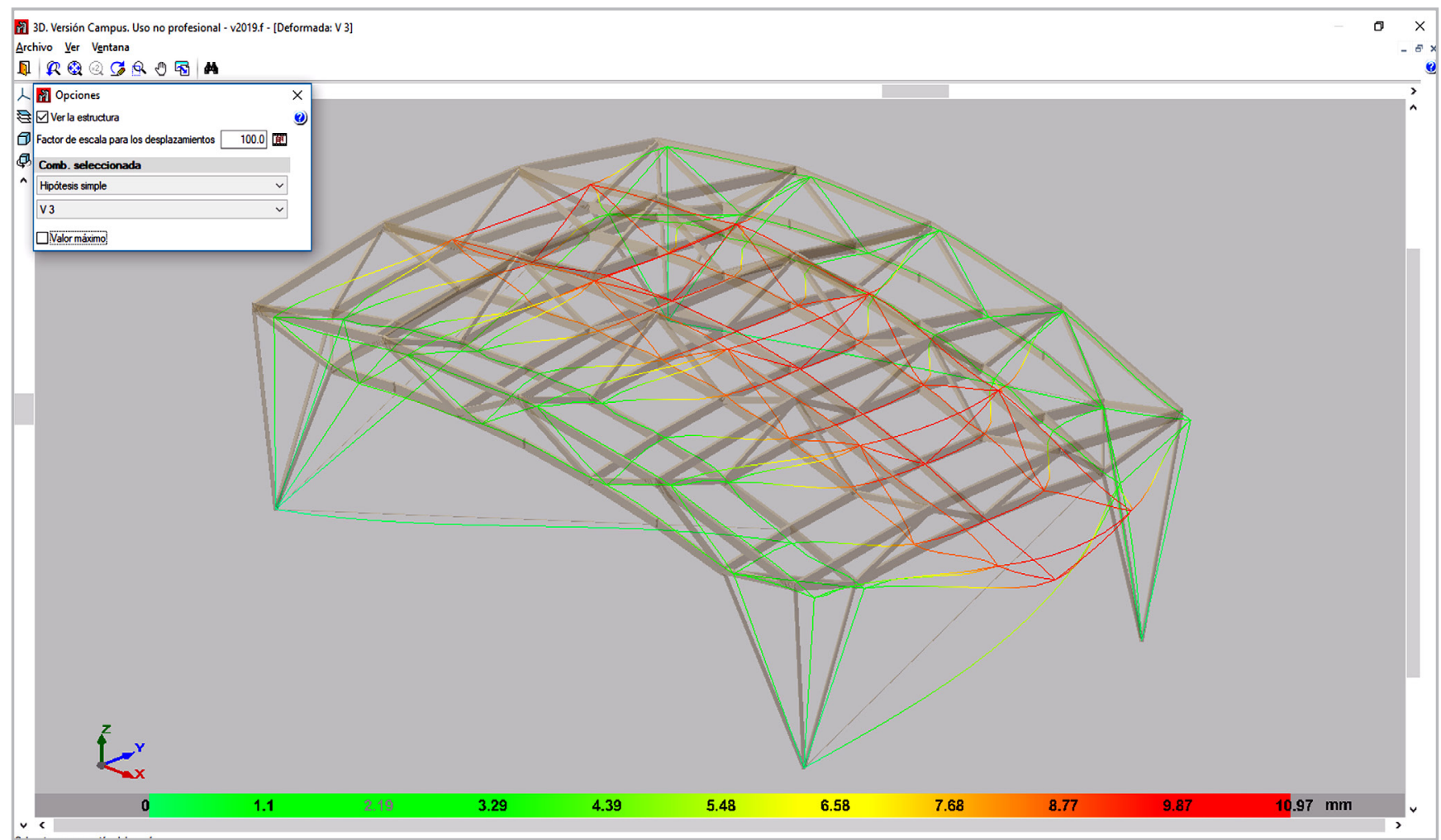

Figura 3.- Salida de resultados de software de cálculo matricial (deformada ante acción eólica).

la acción de viento, [figura 3], considerando si procede la colocación de sistemas ligeros de arriostramiento mediante atirantado, ya que se trata de un sistema de uniones articuladas, consiguiendo con ello apoyos sencillos de escasa incidencia en el suelo.

Como consecuencia del dimensionado estructural, y trasladando los resultados de secciones de barras y nudos al modelo generado paramétricamente, se contará con un modelo virtual con dimensiones reales que puede ser trasladado al sistema de fabricación correspondiente.

Para el caso de estudio, y con el fin de limitar el empleo de material en base a criterios de ligereza y mínima huella ecológica, se opta por el diseño de secciones de barras formadas por dos perfiles separados, unidos puntualmente en el centro de la luz de los mismos, consiguiendo la inercia necesaria. Para el caso de los nudos, se opta por la unión de dos piezas de forma directa atornilladas, lo cual confiere rigidez al mismo, y facilita la unión con el sistema de barras [figura 4].

Finalmente, la generación paramétrica del modelo, y la obtención de un modelo físico digital, permite la fabricación del prototipo de cubierta del yacimiento mediante sistemas de fabricación industrializados, garantizando unos cortos tiempos de fabricación con alta exactitud dimensional de los elementos. Por otra parte, permitirá el rápido trasporte y montaje del mismo, ya que se trata de sistema modular ensamblable configurado para unas condiciones de contorno concretas.
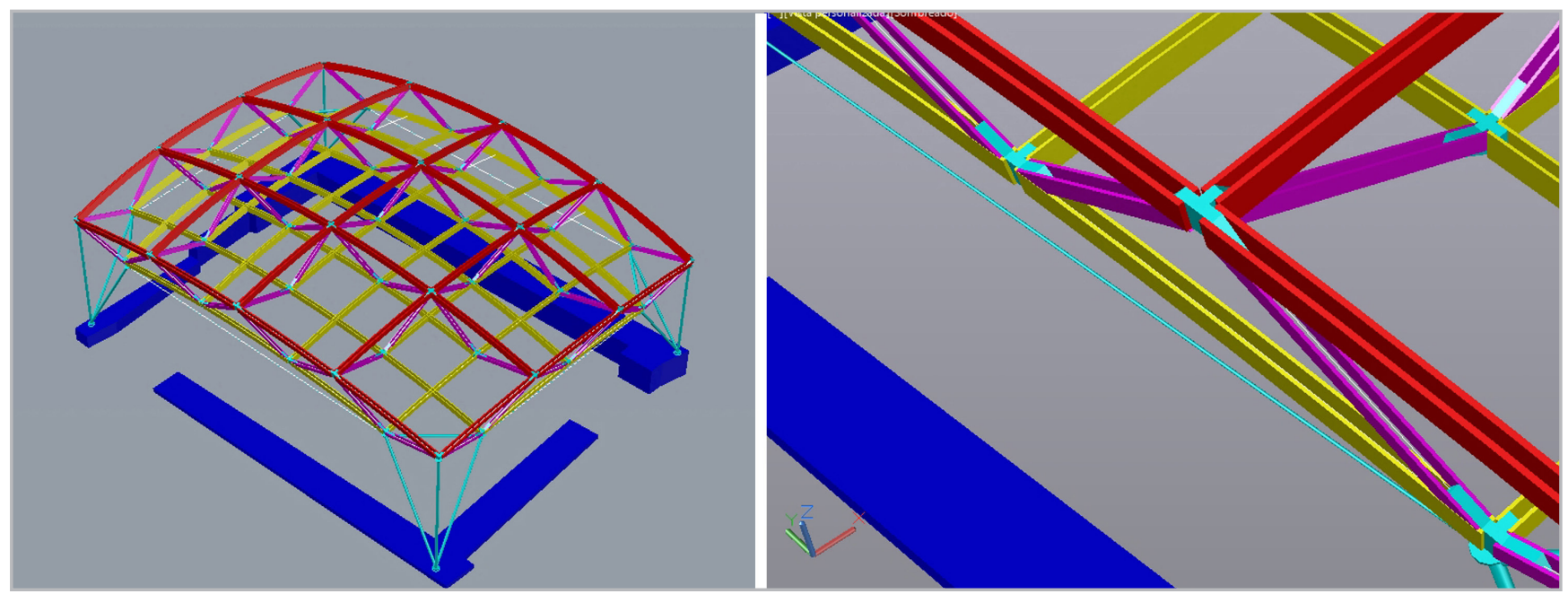

Figura 4.- Evolución del modelo alámbrico al modelo físico dimensionado. 

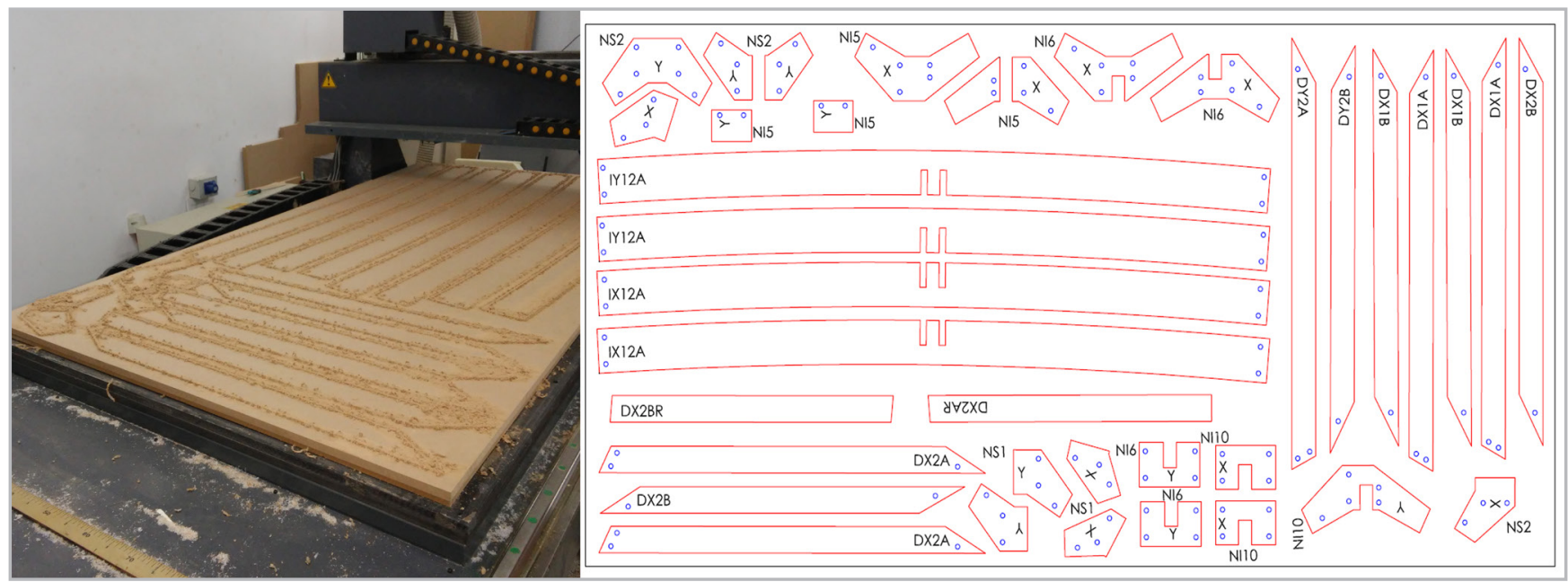

Figura 5.- Panel de despiece codificado y proceso de fabricación en fresadora de control numérico.

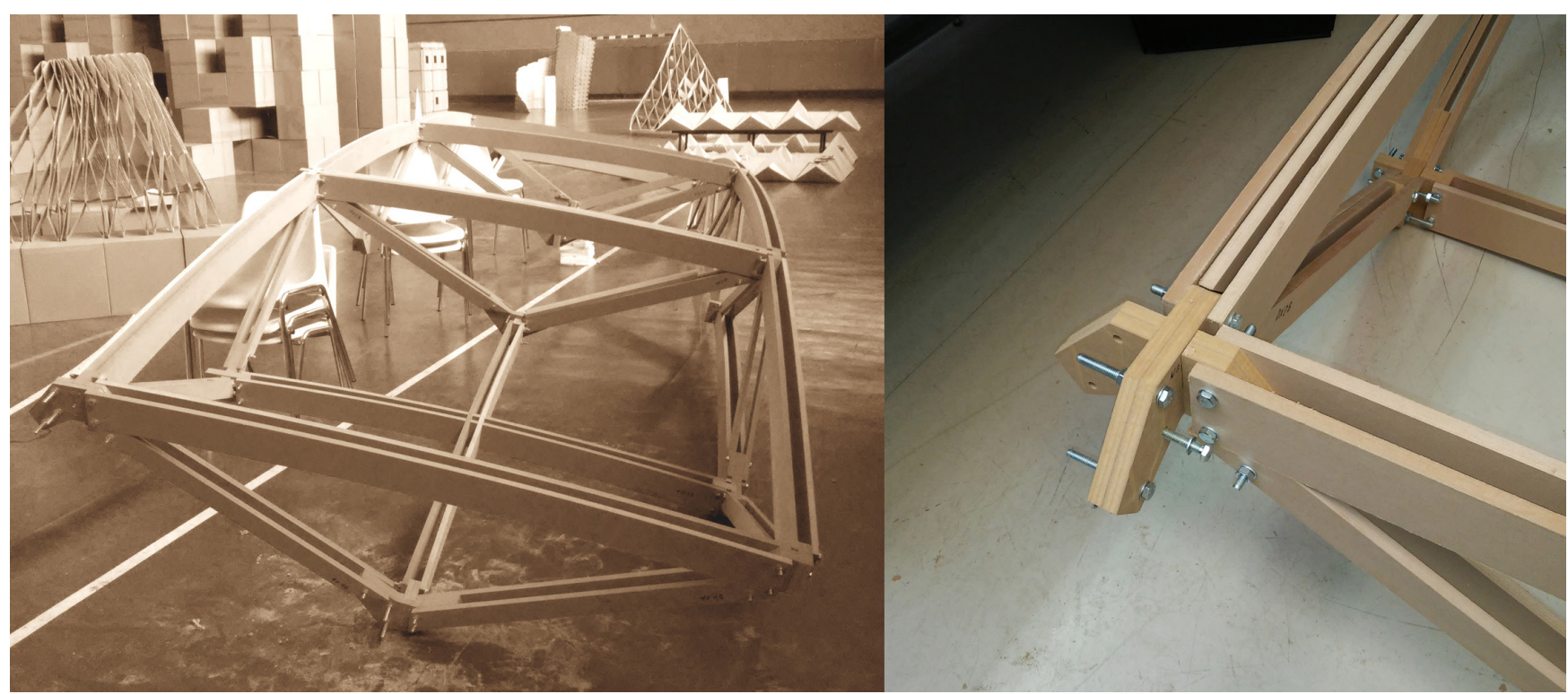

Figura 6.- Fase de montaje de tres módulos de cubierta en taller/ detalle de unión atornillada en cara superior.

La prefabricación en taller de elementos modulares, permite igualmente realizar las correcciones del modelo necesarias debidas a modificación de parámetros durante las labores previas a los trabajos de montaje, incluso debidas a necesidades surgidas durante el proceso de rehabilitación, permitiendo en tiempo real, la modificación de parámetros geométricos de la cubierta (luz, número de módulos, canto de la mallas, etc.), e incluso la extensión de la misma en casos de ser necesario, realizándose la modificación de parámetros en la herramienta de generación, y la nueva obtención del modelo físico digital para su envío a taller.

Como sistema de fabricación, se opta por el empleo de equipo de fresado por control numérico (CNC). Dicho equipo, propio de la pequeña industria de taller metálico y de carpintería, permite el empleo de pequeños recursos con gran economía de fabricación. En el caso de este estudio, se ha empleado el equipo de fresado existente en el Taller de Fabricación Digital de la Escuela Técnica Superior de Arquitectura de Sevilla.
Para la fabricación se han empleado paneles de tablero de densidad media (MDF), de $20 \mathrm{~mm}$ de espesor, con dimensiones adaptadas al equipo de fabricación (1,22 $\mathrm{x}$ 2,44 m). El modelo digital, ha sido despiezado en paneles codificados que son interpretados por la tecnología CNC. La codificación permitirá su posterior instalación en obra como kit de montaje [figura 5].

Con el fin de chequear el proceso, se ha realizado un test de montaje de un primer prototipo en taller, permitiendo la verificación de la coordinación dimensional de barras y nudos, así como la calibración de tolerancias dimensionales en el montaje de las uniones atornilladas. Igualmente, se ha realizado chequeo de estabilidad de los módulos ante solicitaciones previstas, así como ante fenómenos de segundo orden como es el caso del pandeo local de barras, previéndose los rigidizadores necesarios. Igualmente se han instalado los elementos para el futuro anclaje y tensado de las láminas textiles estructurales que constituirán la envolvente. [figura 6]. 


\section{Simulación mediante CFD}

Paralelamente al proceso de fabricación del prototipo, se ha diseñado una herramienta de cálculo original que permita procesos de simulación energética. El estudio tiene como objetivo, elaborar un método analítico que permita documentar la capacidad de las envolventes de doble capa previstas como material de cobertura en yacimientos, para garantizar un control efectivo de variables ambientales mediante sistemas pasivos de mínimo consumo energético.

Será pues necesario, para la aplicación de la metodología de conservación preventiva basada en: diagnóstico, cuantificación de agentes potenciales, estimación de riesgos, proposición de medidas correctoras y monitorización (Carrera 2018), la posibilidad de cuantificar y modelizar los siguientes parámetros ambientales, aplicando un método microclimático (Baglioni; Cacace; Valpuesta 2018):

- Temperatura seca exterior.

- Temperatura seca en el interior del espacio cubierto y en el interior de la cámara de aire formada por la envolvente de doble capa.

- Temperatura superficial de la envolvente y del objeto a proteger (mosaico).

- Humedad relativa interior en espacio cubierto y cámara de la envolvente.

- Velocidad del aire exterior, en interior de la cámara y en interior del espacio cubierto.

- Radiación solar incidente sobre la cubierta.

Para la citada simulación, se ha desarrollado una herramienta de cálculo basada en software de código abierto (PDE), concretamente se empleará la aplicación FreeFem (Chacón 2010), consistente en software para resolver ecuaciones diferenciales empleando método de elementos finitos (FEM).

El modelo de cálculo parte de la definición de límites o bordes cuyos nodos de contacto comparten valores idénticos, permitiendo generar mapas de evolución de parámetros higrotérmicos mediante ecuaciones de continuidad, empleando la dinámica de fluidos computacional (CFD).
Los límites o bordes serán los correspondientes a las diversas capas de textil estructural formado por entramado de fibra de poliéster y protección de polifluoruro de vinilideno de $0.78 \mathrm{~mm}$ de espesor que constituyen la envolvente de doble capa prevista, y que envuelve a la estructura soporte del prototipo. Tendremos pues cuatro límites o bordes que encerrarán las áreas de cálculo (bordes interior y exterior de cubierta, y bordes interior y exterior de envolvente vertical) [figura 7].

La densidad de la malla de nodos y la discretización de las áreas o dominios de cálculo se ajustará a la geometría y a la necesidad de definición de puntos singulares como son las aberturas de admisión-extracción de aire previstas en el modelo [figura 8].

La herramienta diseñada, permite la simulación de variación de parámetros como Ta y humedad relativa, mediante actuación sobre la velocidad del aire en la cámara que forman las dos capas de la envolvente, partiendo de parámetros de condiciones ambientales: Ta exterior, húmedas relativa, radiación solar incidente, velocidad del viento, etc., así como de las características propias de la envolvente (transmitancia térmica, trasmisión, reflexión y absorción solar, factor solar y porcentaje de paso de la luz).

Se ensayan en el modelo de flujos, diversas opciones de admisión y extracción de aire forzado mediante pequeños ventiladores en línea axiales [figura 7], disponiendo aberturas en puntos singulares (envolvente vertical y cubierta), según hipótesis prediseñadas y a ejecutar en el futuro modelo físico a escala 1:1. Detallamos a continuación algunas hipótesis consideradas:

- H1: Admisión forzada unilateral en envolvente vertical y extracción libre en extremo contrario de dicha envolvente.

- H2: Admisión forzada bilateral en envolvente vertical, y extracción libre en envolvente de cubierta.

- H3: Admisión forzada unilateral en envolvente vertical y extracción forzada en extremo contrario de dicha envolvente.

- H4: Admisión forzada bilateral en envolvente vertical, y extracción forzada en envolvente de cubierta.

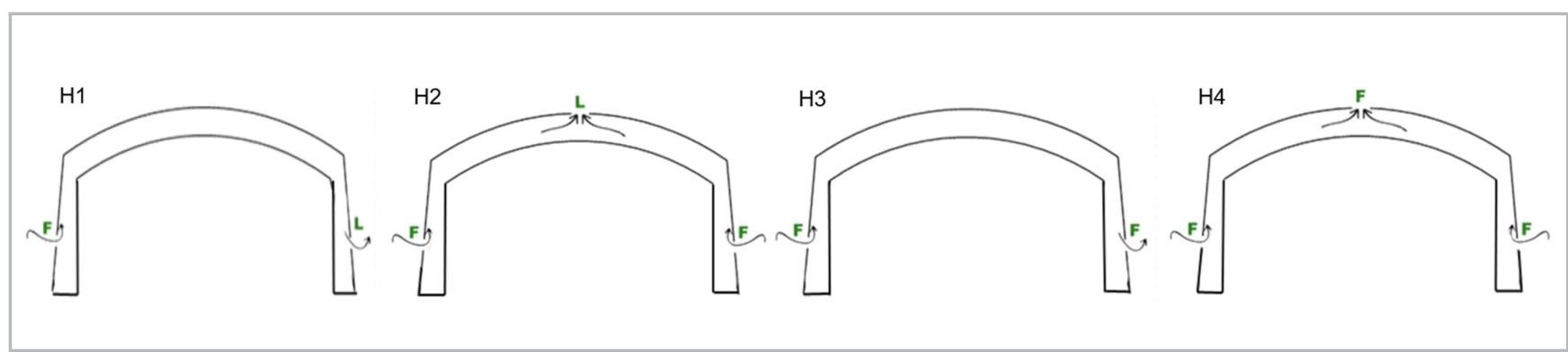

Figura 7.- Esquema de bordes e hipótesis de flujos de aire forzados (F), y libres (L). 


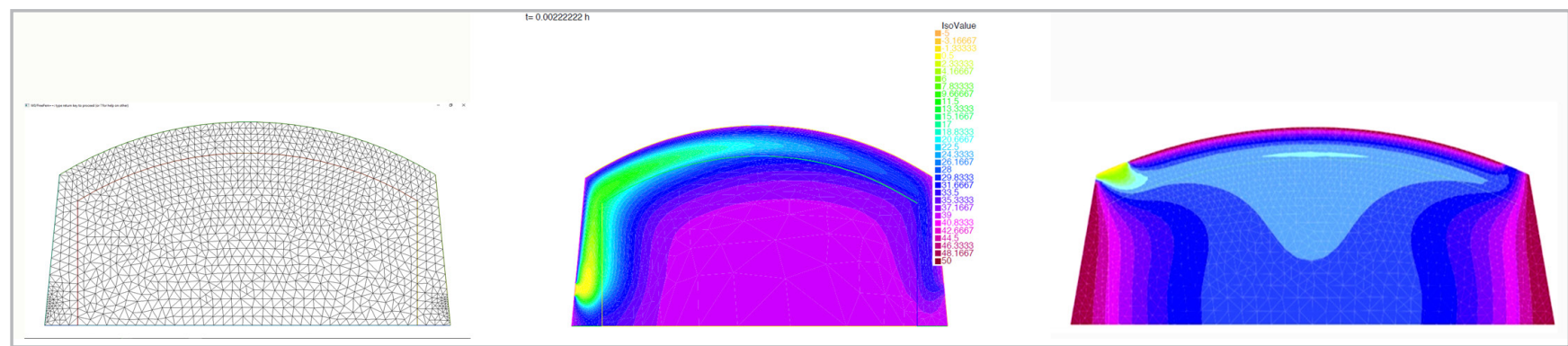

Figura 8.- Modelo de malla discretizada, y modelos de mapas de Ta y velocidad del aire para hipótesis forzada.

Para cada hipótesis, el modelo reporta mapas de evolución estacional y horaria de distribución de temperatura y velocidad de aire, tanto en espacio cubierto como en cámara, ante los diversos flujos estáticos (únicamente debidos a fenómenos de convección por diferencia de temperatura en el espacio cubierto), y ante flujos dinámicos (con circulación de aire forzada en el interior de la cámara formada por la envolvente de doble capa, con diversidad de posición de aberturas [figura 8].

\section{ACV de la solución propuesta}

Tal como se ha indicado, este trabajo se enmarca en una línea de investigación que vincula el diseño del elemento arquitectónico a su influencia en la minimización de su impacto medioambiental en todas las fases de su ciclo de vida (Gómez de Cózar, 2019).

El siguiente cuadro ilustra el modelo arquitectónico utilizado y su relación con las diferentes fases del ciclo de vida de una edificación a efecto de minimizarlas.

En un trabajo anterior (Gómez de Cózar, 2017) se procedió

$\begin{array}{lll}\text { FABRICACIÓN } & \longrightarrow & \text { LIGEREZA } \\ \text { CONSTRUCCIÓN } & \longrightarrow & \text { RÁPIDO MONTAJE } \\ \text { USO } & \longrightarrow & \text { ESTRATEGIAS PASIVAS } \\ \text { ENERGÍAS RENOVABLES } \\ \text { DEMOLICIÓN } & \longrightarrow & \text { REVERSIBLE } \\ \text { DISPOSICIÓN FINAL } & \longrightarrow & \text { RECICLABLE }\end{array}$

a comparar el impacto medioambiental, mediante herramientas de ACV, de una cubierta resuelta con el Sistema Florín frente a otros sistemas cotidianos y usuales que tenían las mismas dimensiones y cubrían la misma superficie. En el estudio reseñado, la estructura de la cubierta Florín, estaba resuelta de forma íntegra en acero galvanizado. En este artículo, se actualiza el análisis en función de la materialidad elegida para la estructura (fundamentalmente, paneles de MDF y tornillería de acero zincadas) asumiendo que todos los modelos que se comparan tienen la misma envolvente (doble capa de membrana textil de malla y urdimbre del poliéster revestida con PVC).

El análisis se ha realizado conforme a ISO 14040 (UNE-EN ISO 14040 2006), ISO 14044 (UNE-EN ISO 14044 2006) y
EN 15978 (UNE-EN 15978 2012), siguiendo el siguiente procedimiento:

\section{Alcance y objetivos (Scope and goal definition)}

El objetivo fundamental en todos los casos analizados ha sido determinar el impacto medioambiental de la edificación proyectada. Siguiendo la bibliografía más actual (Galán Marín et al. 2015), (Asdrubali, F. et al. 2017) se ha considerado el Riesgo de calentamiento global (GWP), como el indicador de impacto más relevante.

\section{Límites del sistema}

En función de EN 15978 (UNE-EN 15978 2012) se han seleccionado las fases más relevantes para el análisis del ciclo de vida de una edificación:

- Fase de producción. Incluye materias primas (A1), transporte de materiales a fábrica (A2) y fabricación de materiales (A3).

- Fase de construcción/deconstrucción. Incluye transporte a lugar de construcción/montaje/obra (A4), proceso constructivo (A5), proceso de de-construcción (C1) y transporte a lugar de disposición final (C2).

- Fase de final de vida. Incluye procesado de residuos para reúso, recuperación y/o reciclado (C3) y disposición final (C4).

En todos los casos se ha considerado una duración de la edificación de 50 años.

Las etapas B1 a B7, relacionadas con fase de uso (energía operacional) no se van a incluir en el presente estudio porque, gracias al uso de estrategias pasivas de diseño, su impacto es despreciable.

\section{Asignación}

Se ha elegido un escenario de reciclado de todos los materiales. Los impactos unitarios asociados a esta fase se han obtenido de la base de datos Ecoinvent 2.0 (Frischknecht, R et al. 2007). 


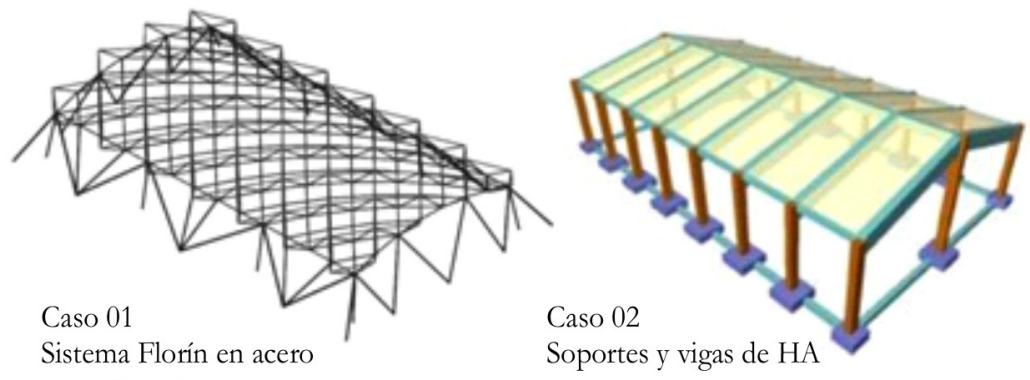

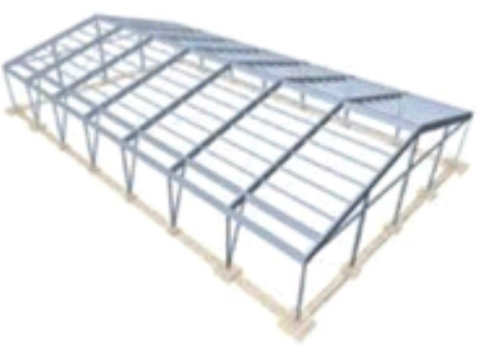

Caso 03

Estructura desmontable de aluminio

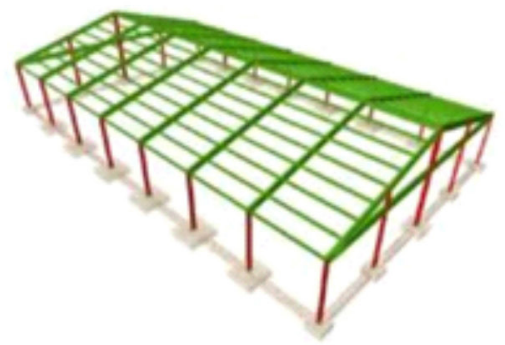

Caso 04

Soportes y vigas

convencionales de acero

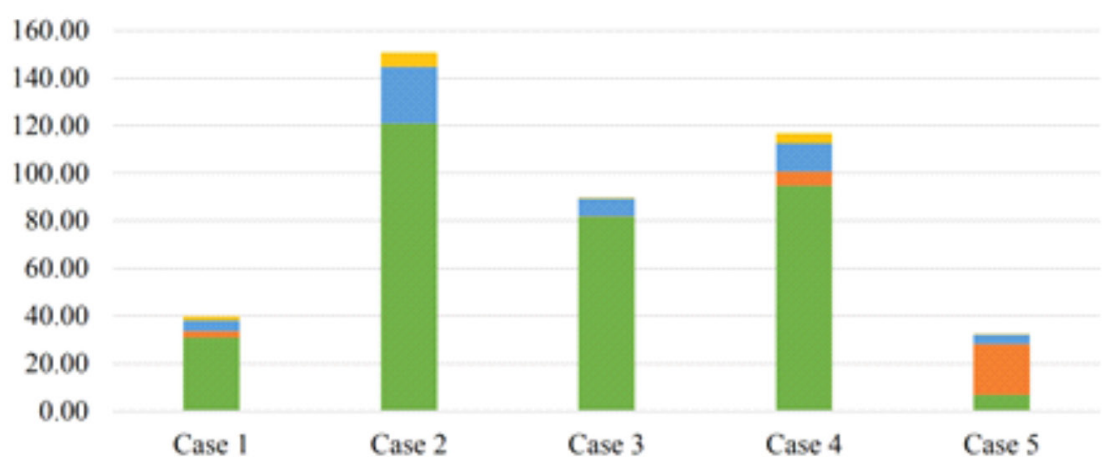

GWP (kgCo2-eq/m2)

\begin{tabular}{lccccc} 
& Case 1 & Case 2 & Case 3 & Case 4 & Case 5 \\
\hline Production & 30,85 & 120,86 & 81,83 & 94,69 & 6,80 \\
\hline Workshop & 2,74 & 0,00 & 0,13 & 5,94 & 21,46 \\
\hline Const.Disassem. & 4,62 & 23,88 & 7,37 & 11,93 & 3,97 \\
\hline End of Life & 1,48 & 6,02 & 0,37 & 4,29 & 0,19 \\
\hline Total & $\mathbf{3 9 , 7 0}$ & $\mathbf{1 5 0 , 7 6}$ & $\mathbf{8 9 , 7 0}$ & $\mathbf{1 1 6 , 8 6}$ & $\mathbf{3 2 , 4 2}$
\end{tabular}

Gráfico 2.- Evaluación del impacto del ciclo de vida. Modelos comparados y resultados.

\section{Unidad funcional}

En todos los casos se ha considerado como unidad funcional el sistema constructivo/estructural completo incluyendo su cimentación. En todos los casos, los resultados de expresan por metro cuadrado de edificación cubierta.

\section{Limitaciones y suposiciones}

Para la cuantificación de la energía demandada durante las fases de construcción y deconstrucción se han cuantificado todos los procesos. En todos los casos se ha considerado un escenario de disposición final de reciclaje de todos los productos empleados.

1. Inventario del ciclo de vida (life cycle inventory, LCI): Consiste en la cuantificación de materiales, procesos y elementos incluidos en el sistema según ISO 14044 (UNE-EN ISO 14044 2006). Se siguen las indicaciones de García Martínez (García Martínez, A. 2010), clasificando el inventario en tres fases:

- Inventario de materiales. 
- Inventario de transportes y procesos de construcción y deconstrucción.

- Inventario de procesos de final de vida.

Los valores de impacto asociados a transportes, construcción y deconstrucción se obtienen a partir del inventario de procesos que se han seguido en las operaciones referenciadas y de los valores de impacto unitarios asociados a cada proceso.

2. Evaluación del impacto del ciclo de vida (life cycle impact assessment, LCIA): A partir de la cuantificación de materiales y de procesos, la evaluación del impacto del ciclo de vida se ha calculado a partir de los diferentes valores unitarios de impacto, según la categoría considerada, establecidos en la base de datos Ecoinvent 2.0. Siendo esta la base de datos de uso más extendido entre la comunidad científica europea. Contiene los valores de impacto asociados a los materiales, elementos y procesos en cada etapa de su ciclo de vida.

3. Interpretación de resultados: En cada caso se han interpretado los resultados estableciendo relaciones entre el modelo arquitectónico/constructivo planteado y los diferentes valores de impacto medioambiental obtenidos.

Los resultados se han repercutido por metro cuadrado de superficie edificada: Modelos 1, 2, 3 y 4:610,00 m²; Modelo 5: 40,32 $\mathrm{m}^{2}$.

Conforme a los criterios establecidos en el punto anterior, comparando los resultados obtenidos con tres sistemas diferentes y también utilizados con frecuencia (estructura de hormigón armado, estructura de aluminio y estructura de acero laminado), los resultados obtenidos ponen de manifiesto que el diseño realizado es el que más minimiza el impacto medioambiental respecto a las opciones restantes. En el gráfico 2 se observan los resultados obtenidos.

\section{Discusión de resultados}

Tras los primeros procesos de generación paramétrica, simulación estructural, higrotérmica y de análisis de ciclo de vida, del modelo, podemos realizar el siguiente análisis:

- Desde el punto de vista del diseño, optimización y comportamiento mecánico: En la optimización estructural, se han seguido criterios de diseño y elección de perfiles basado en: elección de secciones con inercia suficiente y mínimo consumo de material que garanticen la ligereza del sistema, unificación de secciones que permitan fabricación y montaje de mínima complejidad, o uniones entre elementos de rápido montaje y desmontaje, obteniendo como solución óptima la compuesta por trípodes de apoyo a base de perfiles tubulares de acero, y malla de cubierta a base de perfiles dobles de tablero MDF de igual sección en la totalidad de los módulos, previéndose perfil triple únicamente en una barra en los módulos de apoyo en los trípodes. La citada solución supone un peso total de la estructura de $226,50 \mathrm{~kg}$, con una repercusión por superficie cubierta de $5,61 \mathrm{~kg} / \mathrm{m}^{2}$.

Realizando la comparativa con opciones de fabricación en materiales tradicionales de la construcción ligera como el acero y el aluminio, se obtiene una masa total de 500,90 Kg en caso del empleo de perfiles huecos tubulares de sección circular, y de 299,23 Kg en caso de emplear perfilería tubular de aluminio extruido, suponiendo una repercusión de 12,42 y $7,42 \mathrm{~kg} / \mathrm{m}^{2}$ respectivamente, revelándose la solución elegida como la óptima para los parámetros de partida.

-En cuanto a control higrotérmico en el espacio cubierto y objeto arqueológico: Tras la primera fase de simulación, con las hipótesis de aperturas y forzado de flujos de aire descritos [figura 8], podemos analizar los primeros resultados, destacando los siguientes:

En cuanto a valores obtenidos en periodo estival, se observa que el movimiento de aire en el interior de la cámara de la envolvente, permite la disminución de la temperatura interior del espacio cubierto entre 0,5 y $4{ }^{\circ} \mathrm{C}$, y en torno a $2^{\circ} \mathrm{C}$ a nivel del suelo (objeto excavado), para velocidades iniciales de impulsión de aire en la cámara de $13 \mathrm{~m} / \mathrm{s}$, y un periodo máximo de funcionamiento del sistema de 4 horas, obteniéndose lo valores máximos de atenuación para la hipótesis H4 [figura 8], siendo forzada tanto la admisión por envolvente vertical, como la extracción por cubierta, consiguiendo una distribución más uniforme de Ta interior del espacio cubierto, así como una velocidad de aire residual más elevada en el interior de la cámara, en torno a $6,5 \mathrm{~m} / \mathrm{s}$.

En lo que se refiere al periodo de invierno, las primeras simulaciones han revelado que el mantenimiento de velocidades de aire bajas (inferiores a 0,50 m/s según escala de Beaufort), mediante el cierre de aberturas de la envolvente, permite que no exista disminución de la Ta debida a efectos eólicos en el interior de la cámara, favoreciendo el recalentamiento del aire ocluido en la misma debido a la radiación solar directa, con la consiguiente trasmisión de calor a través de la capa textil interior, y el mantenimiento de la Ta seca del espacio cubierto en valores positivos en dicho periodo.

- Relativas a minimización del impacto medioambiental en función de su comparación a otros sistemas constructivos: Como se puede observar en el gráfico 2, las diferentes soluciones que se han comparado aumentan el impacto medioambiental 1,21 veces (caso 1, Sistema Florín resuelto en acero), 4,54 veces (caso 2, hormigón armado), 2,77 veces (caso 3, aluminio) y 3,57 veces (caso 4, acero laminado) respecto a la solución diseñada (caso 5).

Las causas fundamentales de la reducción de impacto son: 
-Naturaleza de los materiales elegidos. Las propiedades naturales del material elegido y su mínima industrialización plantean un potencial mínimo de impacto unitario. La ventaja de poder conseguir cantidades suficientes en un radio de acción cercano minimiza los impactos asociados a transportes.

-Extrema ligereza de la solución. Gracias al diseño de la malla espacial, en función del proceso de optimización que se ha seguido, se consigue un elemento muy ligero. La ligereza repercute en las tareas de fabricación, transportes y montaje.

-Se trata de un sistema de rápido montaje que potencia el trabajo en taller y minimiza el trabajo en obra.

-El sistema es reversible y reciclable al 100,0\%.

Cuando se comparan las dos soluciones (casos 1 y 5 ) que se han resuelto con Sistema Florín, se observa que la solución realizada en madera mejora los resultados que se obtienen con la solución en acero (1,21 veces). Esta situación todavía podría mejorar más en función del tipo de tratamiento que se dé a la madera a efectos de garantizar su durabilidad en ambientes húmedos (clases de servicio 2 y 3 conforme a CTE DB SE M.

De este modo, se pone de manifiesto el beneficio medioambiental que se obtiene al utilizar el modelo constructivo desarrollado en función a otras alternativas posibles.

\section{Conclusiones}

Tras el desarrollo de la metodología propuesta y del análisis de los resultados obtenidos, se concluye lo siguiente:

- El uso de sistemas ligeros y de rápido montaje, como el Sistema Florín, construidos a partir de materiales adecuados, se configura como la opción más idónea de cobertura de yacimiento. La elección de materiales en cada caso de estudio será fundamental atendiendo no sólo a la naturaleza de éstos sino también a su necesidad de ser protegidos con otros productos para garantizar su durabilidad. Las herramientas de diseño paramétrico creadas, unidas a la fabricación digital, permiten obtener soluciones originales y optimizadas en cada caso en función de las necesidades particulares de cada yacimiento. En cada caso, aunque el sistema sea el mismo, la geometría final estará adaptada a las condiciones reales del yacimiento.

- La incorporación de doble capa de membrana a un sistema constructivo que fue creado con ese fin, permite tener un control higrotérmico aceptable del espacio cubierto sin apenas consumo energético, empleado medidas pasivas de acondicionamiento ambiental.

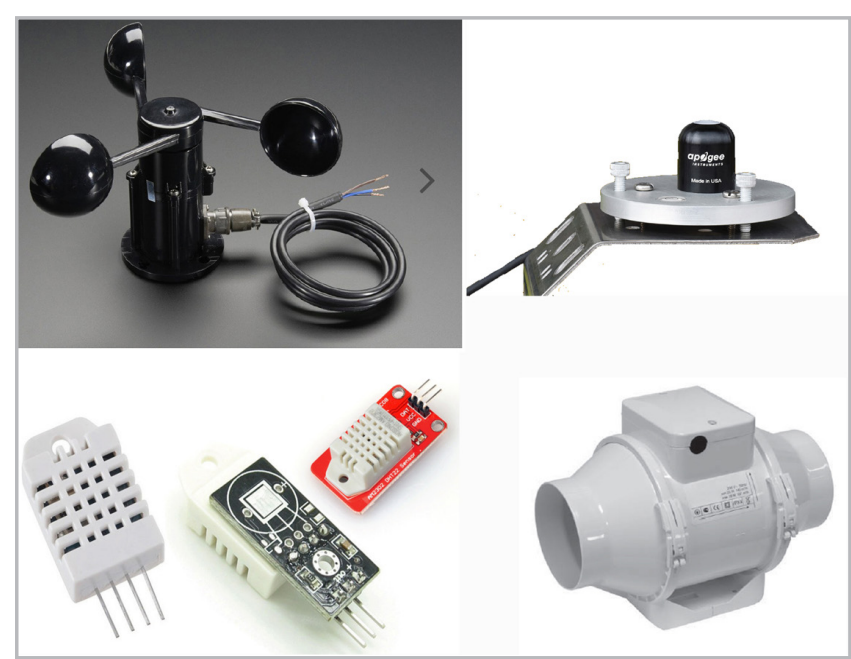

Figura 9.- Sondas y equipamiento a instalar en el prototipo construido.

Las simulaciones que se han realizado a partir de las herramientas originales creadas ponen de manifiesto el beneficio de esta tecnología a la hora de conseguir temperaturas adecuadas tanto en el interior del recinto como en su suelo.

- Se ha demostrado, como los dos puntos anteriores tienen gran repercusión en el Análisis del Ciclo de Vida realizado en cuanto a la minimización del impacto medioambiental conseguido. Cuando se sigue la metodología propuesta, se ha podido comprobar que el elemento resultante es el que menos impacto produce en relación a otros sistemas habituales, en las mismas condiciones de uso, con los que se ha comparado.

\section{Desarrollos posteriores a la redacción de este artículo. Futuras líneas de investigación}

Dentro del estudio del prototipo, y una vez sea instalado en el emplazamiento propuesto, se procederá a la monitorización del mismo, con el fin de contrastar los datos de simulación del modelo de cálculo CFD. Para ello, se recurrirá a tecnología compatible con software de código abierto "Arduino" (Evans 2007), colocándose sondas de temperatura y humedad en el exterior del mismo, en interior de cámara de la envolvente textil, y en el interior del espacio cubierto. Igualmente se prevé el uso de dinamómetro y piranómetro, para obtener lectura de parámetros exteriores de velocidad del aire y radiación solar incidente, así como de pequeños equipos de ventilación que permitan modificar la velocidad del aire en la cámara, e incluso la trasferencia de aire de la misma al espacio interior [figura 9]. La comparación de los datos obtenidos por el conjunto de sondas y equipamiento permitirá la gestión de una gran base de datos de parámetros de condiciones ambientales del espacio cubierto ante la variabilidad de las condiciones exteriores estacionales, y las condiciones activas de intervención 
sobre el mismo, permitiendo así la calibración del modelo de cálculo.

\section{Referencias}

AA.VV. (2008). Deplorable Emergency System. La Coruña: Netbiblo S.L.

AA.VV. (2003). Taller de Conservación Preventiva en Excavaciones Arqueológicas in Situ. Cádiz: Instituto Andaluz de Patrimonio Histórico, Colegio Oficial de Doctores y Licenciados en Filosofía y Letras de Cádiz.

ASDRUBALI, F., et al. (2017), "Abrevies of Structure, Termo-Physical. Acoustic, and Environmental Propperties of Wooden Materials for Buiding Applications", Building and Environment, 114: 307-332.

BAGLIONI, R., CACACE, C., VALPUESTA, S. (2018). "Diseño y Evaluación de una Metodología de Estudio y Gestión Medioambiental de Bienes Culturales". En VI Congreso GE IIC, Vitoria-Gasteiz: Grupo Español del IIC: 198-205.

CARRERA, F. (2018). "Conservación Preventiva en Yacimientos Arqueológicos, ¿Empezamos?". En VI Congreso GE IIC, VitoriaGasteiz: Grupo Español del IIC: 376-384.

CHACÓN, E. (2010).“Notas sobre FreeFem++2Dy3D:traducción del manual de F. Hecht, en Departamento de Ecuaciones Diferenciales y Análisis Numérico, Facultad de Matemáticas, Universidad de Sevilla. http://freefem.org/ff++/ftp/freefem++Spanish.pdf

DÍAZ MARTínEZ, S. (2005). “La Conservación de los Yacimientos Arqueológicos In Situ", Boletín Arkeolan, 13: 110-130.

EVANS, B. (2007). Arduino Programming Notebbok. San Francisco: Creative Commons.

FRAZZI, P. (2002). "Conservación preventiva para objetos arqueológicos históricos en contextos urbanos", Estudios Ibero Americanos de la Universidad Pontificia de Rio Grande do S, 26 vol XXVIII: 95-111.

FRISCHKNECHT, R., JUNGBLUTH, N., HANS-JÖRG, A., et. al. (2017). Implementación of life cycle impacto assessment métodos: data v2.0. Dübendorf: Rolf Frischknecht, Niels Jungbluth.

GALÁN MARÍN, C., RIVERA GÓMEZ, C., GARCÍA MARTÍNEZ A. (2015). "Embodied Energy on convencional load-Bea ring salles versus natural stabilized e Art ha blocs", Energy and Building, 97: 146-154.

GARCíA MARTínEZ, A. (2010). Análisis de Ciclo de Vida (ACV) de edificios. Propuesta metodológica para la elaboración de Declaraciones Ambientales de Viviendas en Andalucía. Sevilla: Universidad de Sevilla.

GÓMEZ DE CÓZAR, J. C., GARCÍA DIÉGUEZ, R. (2001). Sistema Florín. Estructuras Desplegables de Dos Capas. Sevilla: DCA1 Universidad de Sevilla.
GÓMEZ DE CÓZAR, J. C. (2007). "Textile Architecture?". En III International Conference on Textile Composites and Inflatable Structures, Barcelona: International Centre for Numerical Methods in Engineering: 293-296.

GÓMEZ DE CÓZAR, J. C., GARCÍA MARTÍNEZ A., ARIZA LÓPEZ, I., et. al. (2017). "Lightweight and Quickly Assembled: the Most Eco-Efficient Model for Architecture", International Journal of Computational Methods and Experimental Measurements, Vol. 5. Núm. 4: 539-550. https://doi.org/10.2495/CMEMV5-N4-539-550

GÓMEZ DE CÓZAR, J. C., GARCÍA MARTÍNEZ A., ARIZA LÓPEZ, I., et. al. (2019). "Life cycle assessment as a decision-making tool for selecting building systems in heritage intervention: Case study of Roman Theatre in Itálica, Spain", Journal of Cleaner Production, 206: 27-39. https://doi.org/10.1016/j. jclepro.2018.09.169

JIANG, HE., AKIRA, HOYANO. (2009). "Measurement and simulation of the thermal environment in the built space under a membrane structure", Building and Environment ,44: 11191127. https://doi.org/10.1016/j.buildenv.2008.08.003

KRAUEL, J. (2010). Arquitectura Digital, Innovación y Diseño. Barcelona: S.A. Leading International Key Services

KRONENBURG, R. (2008). Portable architecture: design and technology. Basel: Birkhäuser.

LACAYO, T.E. (2001). "Factores de Alteración in-Situ. Conservación Preventiva del Material Arqueológico". En XV Simposio de Investigaciones Arqueológicas en Guatemala, Guatemala: J.P. Laporte, H. Escobedo y B. Arroyo: 453-457.

PRICE, S. (1984). La Conservación en Excavaciones Arqueológicas. Roma: ICCROM.

\section{Autor/es}

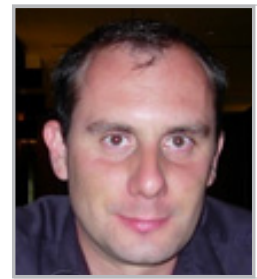

Manuel Ordóñez Martín

maormarq@us.es

Universidad de Sevilla. E.T.S. Arquitectura

Profesor Asociado Universidad. Máster en Arquitectura y Patrimonio Histórico. 2012. Profesor Máster en Proyecto de Instalaciones en Arquitectura. (2011-actualidad). Departamento de Construcciones Arquitectónicas 1. Universidad de Sevilla. E.T.S. Arquitectura. Grupo de Investigación TEP 130: Arquitectura, Patrimonio y Sostenibilidad: Acústica, Iluminación, Óptica y Energía. Doctorando con tesis en redacción: Análisis y Desarrollo de Coberturas Temporales Eficientes en Yacimientos Arqueológicos. Producción científica más relevante. https://investigacion.us.es/sisius/sis showpub.php?idpers=7429 


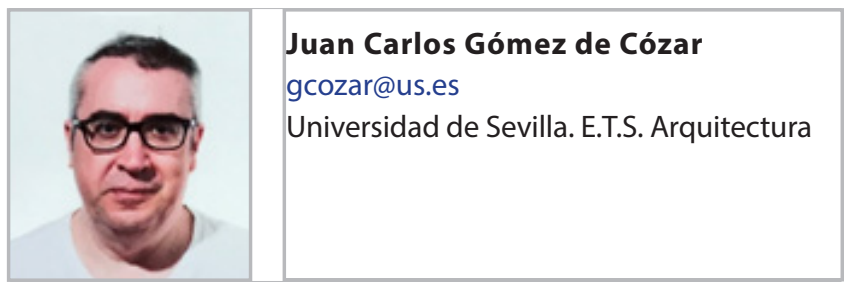

Profesor Titular de Universidad. Director del Master Universitario en Innovación en Arquitectura: Tecnología y Diseño. Departamento de Construcciones Arquitectónicas 1. Universidad de Sevilla. E.T.S. Arquitectura. Grupo de Investigación TEP 130: Arquitectura, Patrimonio y Sostenibilidad: Acústica, Iluminación, Óptica y Energía. Autor de varias patentes y de numerosos trabajos de transferencia que desarrollan la línea de investigación: "Forma, materialidad y arquitectura. búsqueda de modelos constructivos ligeros, de rápido montaje y reversibles que minimicen el impacto medioambiental". Producción científica más relevante:

https://investigacion.us.es/sisius/sis showpub.php?idpers $=5478$

\section{(@) $(\Theta \Theta$}

https://doi.org/10.37558/gec.v17i1.757 